\title{
Intelligent Approach to Minimizing Power Consumption in a Cloud-Based System collecting Sensor Data and Monitoring the Status of Powered Wheelchairs
}

\author{
Ogechukwu Okono, Alexander Gegov, Mo Adda, \\ David A Sanders, Malik Jamal Musa Haddad, Giles Tewkesbury \\ University of Portsmouth
}

\begin{abstract}
This paper proposes an intelligent approach to minimizing power consumption in a cloud-based system collecting sensor data and monitoring the status of powered wheelchairs. The powered wheelchairs will be equipped with sensors and the system collects sensor data to track daily activities and locations. The proposed cloud-based system will allow caregivers real-time access to information generated by the wheelchairs. Cloud computing has emerged as the leading paradigm for information technology. The recent growth of the internet and significant innovation have accelerated the development of many distributed computing schemes and cloud computing has gained wide acceptance. There are still some draw backs though. For example, cloud network power consumption is increasing. This has led to providers increasing the sizes and numbers of data centres. The high-power consumption of each data centre is concerning so an improved method with some level of intelligence is used to minimize power used in the data centre network. Power models are mathematically represented. The mechanism described will be able to regulate activities in a centre and switch-off inactive switches and links. Servers and network activities will be consolidated to make them more energy efficient.
\end{abstract}

Keywords: power consumption, cloud, wheelchair, assist, sensors, network, data, energy, computing, scheduling, quality.

\section{Introduction}

Smart wheelchairs [1-6] are becoming more popular as the number of elderly and disabled people increases. Users need more efficient care services [7] and smart wheelchairs can include new technologies such as Body Area Networks (BANs) [8, 9] working with cloud computing [10], sensors [11-22], sensor data fusion [23], wireless communication, and embedded systems [24].

In this paper, new cloud-based systems are considered that can assist wheelchair users by communicating with BANs to obtain data about the users and their wheelchairs. Careers can access the data log for a wheelchair user and provide health advice health and predict disease risk. Additionally, some dangerous situations can be detected, such as a wheelchair overturning, and the system could send an alarm.

Cloud computing is becoming more common. It was initiated in late 2006 by major industry giants (Google, Amazon, etc) [25]. As many proprietary software, platforms and infrastructure continue to increase in price and availability, the need of CC becomes irresistible. With the advent of CC, many firms (especially medium size enterprise) can now enjoy different range of services without having to acquire them and this has encouraged rapid rise in the sector and boom in certain economies. According to the National Institute of Standards and Technology (NIST), CC model should be composed of three service levels (viz: Infrastructure as a Service (IaaS), Software as a Service (SaaS), and Platform as a Service (PaaS)), five characteristics (viz: on-demand self-service, broad network access, resource pooling, rapid elasticity, and measure service), and four deployment models (viz: private, public, community and hybrid clouds) [26]. It is also worth noting that cloud is not the same as data center. While cloud stores data on the Internet, data centers do the same but within the organization's local network. However, cloud service providers use data centers to deploy their services in different geographic locations [27]. Taking a case study by S Mittal et al [28], it showed that more than 100 million videos are watched via YouTube per day, while Facebook has more than 400 million active users and 3 billion photos uploaded every month. All these activities are powered by data centers through their computation-intensive software programs (this underscores the amount of energy usage).

Figure 1 is a systematic representation of cloud data center taxonomy. Data center is classified into two major types: the computing resources (which is the IT equipment) and the Physical resources (which is the infrastructure). Infrastructure as a service basically provides processing power or storage.

According to Steven Pelly [29] published article, it was said that data center's power consumption will continue to triple its growth rate and projected it will reach 100 billion $\mathrm{kWh}$ at an annual cost of $\$ 7.4$ billion within two years. Furthermore, relating this published figure to Google's vendor statement [30], of them alone using up to 2,675,898 mWh on power in 2011 and the breakdown of it goes to server and cooling equipment. In fact, Google also forecast a $10 \%$ increase in power usage too if nothing is done quickly to mitigate the power consumption rate - a good design power management approach is a must have solution to the impending problem. Recently, many studies have focused on the energy consumption levels of data centers [31,32]. The Environmental Protection Agency (EPA) study estimated that the data center's energy consumption would double from 2006 (61 billion kWh) to 2011. In 2009, data centers accounted for $2 \%$ of worldwide electricity consumption with an economic impact of US \$30 billion [33]. The authors of [34] forecasted data center hardware's expenditure to be US \$106.4 billion by 2012, a $12.7 \%$ increase from 2011. Cloud computing revenue is also forecasted by to jump from US \$163 billion in 2011 to US \$240 billion in 2016 . Table 1 is the summary of data center power consumption from 2005 - 2017. 


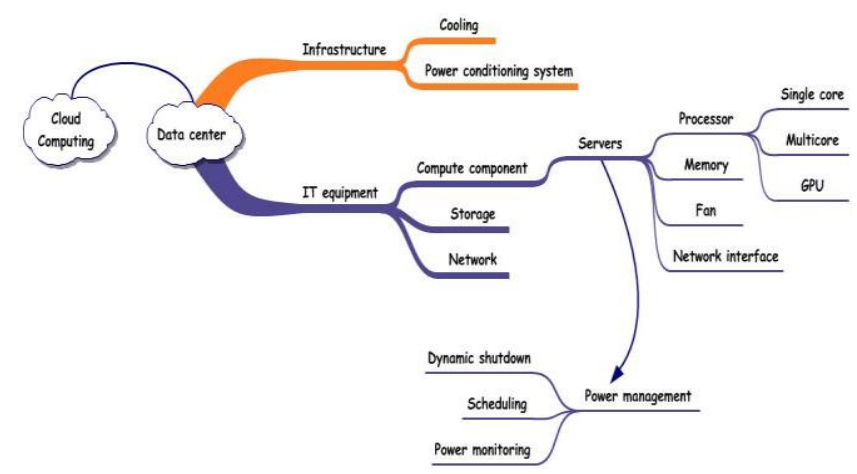

Fig. 1. Taxonomy of cloud data center.

Table 1: Summary of data center power consumption from 2005 - 2016

\begin{tabular}{|c|c|c|c|c|}
\hline Year & Investigator & Cost & KWH rate & \% rate \\
\hline 2005 & Miyuru et al & - & - & $\begin{array}{l}1 \% \text { of } \\
\text { US power } \\
\text { consumption }\end{array}$ \\
\hline 2006 & $\begin{array}{l}\text { R. Brown et al } \\
\text { [2] }\end{array}$ & $\begin{array}{r}\$ 4.5 \\
\text { Billion }\end{array}$ & 61 Billion & $1.5 \%$ \\
\hline 2009 & G.Meijer et & $\begin{array}{r}\$ 30 \\
\text { Billion }\end{array}$ & - & \begin{tabular}{l}
\multicolumn{1}{c}{$2 \%$} \\
worldwide \\
electricity \\
consumption
\end{tabular} \\
\hline 2011 & Google vendor & $\begin{array}{l}\quad \$ 163 \\
\text { Billion }\end{array}$ & $2,675,898$ & - \\
\hline 2012 & Garter & $\begin{array}{l}\text { \$106.4 } \\
\text { Billion }\end{array}$ & - & $12.7 \%$ \\
\hline 2016 & X. Fan et al & $\begin{array}{l}\quad \$ 240 \\
\text { Billion }\end{array}$ & - & $28.9 \%$ \\
\hline 2017 & Garter Report & $\begin{array}{l}\text { \$346 } \\
\text { Billion }\end{array}$ & & $32 \%$ \\
\hline
\end{tabular}

\section{Background}

\subsection{Data Centre (DC) Topology}

This paper considers the best fit data center topology with limited port densities in the highest end commercial switches. The threetier trees topology happens to be the most accepted and suitable type because of its higher-speed links. The three tier tree consist of the core layer which is at the root of the layer, the aggregation layer which is full responsible for routing and the access layer which holds the racks (computing severs). The computing servers are grouped in racks and the connectivity of the racks are achieved with Top-Of-Rack (TOR) switches which can be matched to Point-Of-Presence (POP) in the network. A typical TOR switch shares two 10GE uplinks with 48GE links that interconnect computing servers within the rack. The racks are aligned in modules with a pair of aggregation switches serving the module connectivity. Equal Cost Multi-path (ECMP) routing technology is used to distribute the bandwidth between the core and the aggregation network. Looking at a typical data center diagram in Figure 2 it will be easy to understand the reason behind the complexity of its connection.

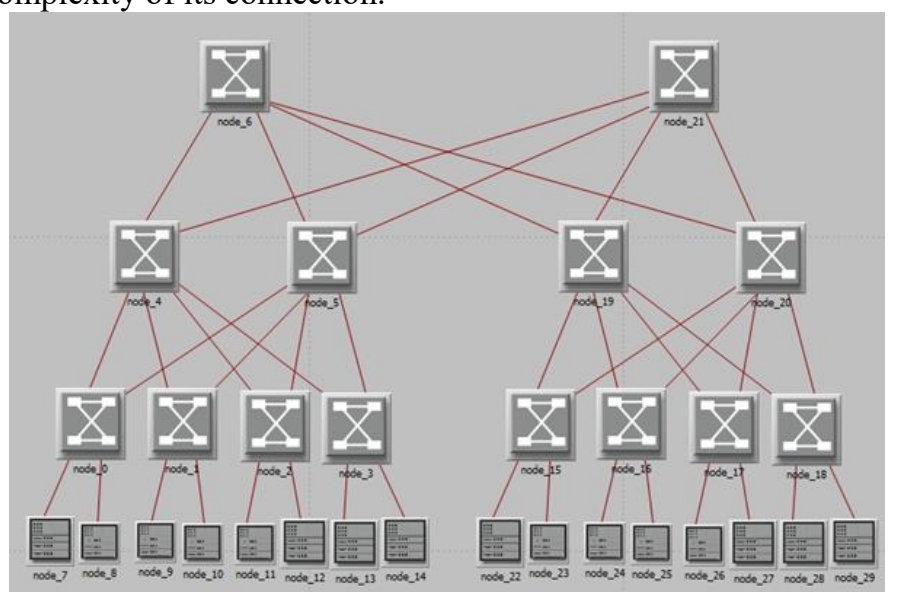

Fig. 2. Data center structure. 
This section is limited to green CC state-of-art techniques which can be classified as: green CC based on, green Internet resources, over-provisioning and scaling of cloud resources, and task scheduling.

\subsection{Green CC based on green Internet resources}

$\mathrm{CC}$ is demand driven i.e. it provides pay-as-you-go services over the Internet. This implies that without Internet, there is no CC. The authors of $[37,38]$ showed that ICT alone contributed between $2-10 \%$ of world's energy consumption, and with a prediction of 20 - 25\% annual growth. This rapid increase in energy consumption of Internet-based networks has become a major concern to both the $\mathrm{CC}$ operators and the $\mathrm{CC}$ customers due to the increasing electricity cost and the environmental effect. In [39], the authors showed that ICT also accounts for $2 \%$ of the greenhouse effect annually. All these effects could be attributed to the increasing demand in traffic as a result of the introduction of high bandwidth requirements and Quality of Service (QoS) measures in new applications. Furthermore, the energy consumption of computing devices remains almost steady once it is powered on i.e. the energy consumption does not scale with the traffic load [40,41]. In recent times, $\mathrm{CC}$ has received maximum attention due to its ability to delivering ICT services by optimizing the data centre facilities. Below in fig 3 is the graphical representation of data centre electricity usage forecast till 2022.

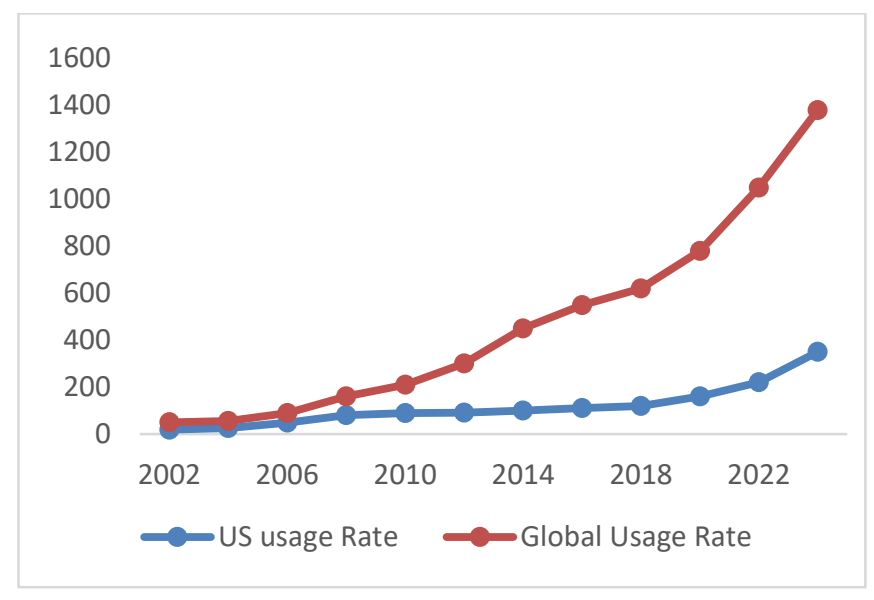

Fig. 3. Graphical representation of data centre electricity usage forecast (Mckinsey)

\subsection{Over-provisioning and scaling of cloud resources}

In view of optimizing the energy consumption of $\mathrm{CC}$ resources while keeping service level from customers point, many methods do exist in the literature as can be seen in [42]. It has been estimated that data centers in United States of America contributed about $1.5 \%$ of the overall energy consumption in that year and this is expected to grow at the rate of $18 \%$ annually [43]. The easiest way is to minimize the number of active machines. However, this may be inimical to sudden increase in traffic demand since network efficiency is the main concern of the customer. This implies that most customers, though worry much on the energy consumption rate, will not sacrifice the efficiency of their service. Some models have shown that the energy consumption can be attributed to different components of the $\mathrm{CC}$ world which includes the nodes, switches, storages, amongst others [44, 45]. This has led to green hardware proposal through rate adaptation $[46,47]$. Rate adaptation can be defined as a hardware mechanism of energy saving by operating a device at lower voltage (i.e. Dynamic Voltage Scaling - DVS) or frequency (i.e. Dynamic Frequency Scaling - DFS). The authors of [48] proposed the deployment of dynamic voltage frequency scaling (DVFS). However, since idle servers consume about two third of the peak and load, the efficiency of DVFS is limited.

The diurnal traffic of a data center is unpredictable and fluctuates in between the peak and off-peak periods. This has led to the massive deployment and over-provisioning of CC resources in order to cope with the traffics. It has been observed by [49] that the average load in data centers is about $30 \%$ of the resources and this underscores the high level of resource over-provisioning. Therefore, reducing these resources in a systematic way that the cloud efficiency is not sacrificed is another research direction and that is the aim of this proposal. Of course, this can save up to $70 \%$ of the energy used. It has also been shown that the IP router's line card energy consumption dominates the total energy of the network to the tune of $43 \%$ of the total power of the router [50, 51]. The authors of [52] proposed the combination of both traffic and work load consolidation schemes. Of course, this work is not the first proposal on energy efficiency on CC but most research do not optimize both the efficiency of CC and its energy savings.

On the issue of accepting the adoption of $\mathrm{CC}$, the authors of [53] explored the factors affecting CC adoption. Using questionnaires-based methodology; they propose sets of different variables for CC adoption and non-adoption. More so, they explored available opportunities for CC service providers. In [1], a classification of CC research approach was carried out. The classification includes business issues, technological issues, domains and applications, and conceptualizing CC. Another research work on classification was done in [54] where they explored the concept of visualization in computing. They also enumerated the motivation behind $\mathrm{CC}$ with review of several cloud deployment and service models. 


\subsection{Task scheduling for green CC}

Deploying a better scheduling approach to leverage cloud data center power consumption rate is very vital to sustaining this emerging technology. In this internet era there has been significant increase in the amount of migration seen in the last few years into the cloud environment. This is good but will require more intelligent scheduling to be able to copy with demands and still maintain the SLA with a good power usage effectiveness. In view of this, it is impossible to discuss cloud data center or cloud environment power usage without first looking at the server's activities and its scheduling procedure. According to the recent work done in literature [55-57] scheduling method has been classified into three groups namely: resource scheduling, task scheduling and workflow scheduling. Considering the complexity of cloud environment this work, will be focusing mainly on task scheduling method. Still under the task scheduling approach, there are three categories mentioned but the interest will be on the heuristic approach which is based on [43], [58], [59]. The authors provide optimal solution because it uses the knowledge base approach to make scheduling decisions. Basically, a scheduling process starts when a user submits its requested task to the cloud system. The task gets to the data center, and then the data center classifies the task according to SLA and requested services. Afterwards each task is assigned to one of the available servers. In turn, the servers perform the requested task and then a response is transmitted back to the user as a result. This scheduling procedure is effective but on the other hand, it still needs to strike a balance trade-off between reliable performance and energy cost. With reference to the literature [25], heuristic task scheduling approach which is considered the best because it takes less execution and completion time, still comes with its disadvantage for it does not consider the available resource at the time of scheduling, thereby causing load unbalancing.

Most of the above research papers centered on just scheduling the jobs with no added features, some focused-on network traffics and server consolidation with less emphasize on how to achieve more efficient power used system with less complexity. A hybrid approach with more energy efficient technique will balance the pressure between efficiency of service versus efficiency of power used. However, this work considers a holistic approach which comprises the network traffics, links, and nodes. Other proposals have only succeeded in taking one into action and ignoring the other; this does not optimize best result.

\section{$4 \quad$ Algorithms}

\subsection{Proposed mathematical formulation of Energy model in DC}

Elaborating in detail how the components of cloud DC works will give a clear understanding of what to expect when calculating the energy models involve. Looking at the sample typical DC topology in fig. 2 which consist of one core switch, two aggregation switches and four access servers. Table 2 have the fully description of the variable used in formulating this energy model.

Table 2. List of Symbols.

\begin{tabular}{|c|c|}
\hline Variable & Description \\
\hline$P_{i}$ & Ports \\
\hline$P$ & Power \\
\hline $\mathrm{E}$ & Unergy \\
\hline$\mu$ & Server \\
\hline$S_{r v}$ & Rate \\
\hline $\mathrm{R}$ & set of network device \\
\hline $\mathrm{Nc}$ & set of links \\
\hline $\mathrm{V}$ & Switches \\
\hline$\varepsilon$ & Computing cost \\
\hline$S_{w i}$ & . \\
\hline$P_{c c}$ & \\
\hline
\end{tabular}

In this paper we will be calculating the total Power consume in the DC which includes the summation of the total computing power (both the computing and networking device) which are sometimes overlooked in other articles but will help give a better view of how minimizing its activities can contribute to energy efficient data center. Therefore, the total computing cost will:

$$
P_{c c}=P_{s r v}+P_{s w i}
$$

Now, knowing that switches form the basis of the interconnection fabric that delivers task requests to the hosts for processing. Energy consumption of a switch depends on the following: number of ports, port transmission rate, types of switch and the deployed cabling solution based on the simple 3tie or fat tree topology in fig 2 which this formulation is based on. The energy consumption model for switches then: 
Where

$$
P_{\text {swi }}=P_{\text {chassis }}+n_{\text {linecard }} . P_{\text {linecard }}+\sum_{i=0}^{c} n \text { ports. } P_{r} * \text { UtilizationFactors }
$$

$P_{\text {chassis }}:$ The power consumed by the switch base on hardware

$P_{\text {linecard }}:$ The power consumed by the active line cards

$P_{r}$ : The power consumed by the active port running at ' $r$ ' rate

Computing Server also contributes heavily to the high energy consumption rate in the data center. It will be ideal to say that the energy consumption of a host server is proportional to the CPU utilization. Previous research has shown that an idle server consumes two-thirds of its peak-load consumption to keep memory, dish and input output resources running. Its energy model is given as [35]:

$$
E(A)=E_{c p u}(A)+E_{\text {memory }}(A)+E_{\frac{I}{0}}(A)
$$

Building on this, the energy model for cost of power used by the server will then be

$$
P=P_{s r v}-\sum_{j=1}^{n} \delta^{j} P_{j}
$$

Where $\delta_{j}=\left\{\begin{array}{r}0 \quad \text { if server } j \text { is enable } \\ 1 \quad \text { if server } j \text { is disable } \\ \text { fi if server } j \text { is in standby }\end{array}\right.$

Which can also be represented as $0 \leq \delta_{j} \leq 1$

Then

$$
P=\sum_{j=1}^{n} P^{\wedge} j-\sum_{j=1}^{n} \delta^{j} P_{j}=\sum_{j=1}^{n}\left(1-\delta_{j}\right) P_{j}
$$

Therefore, to calculate the total power cost of the cloud computing device will refer to equation 1

Which will then be

$$
\begin{aligned}
& \sum_{j=1}^{n}\left(1-\delta_{j}\right) P_{j}+\sum_{\substack{n=1\\
}}^{m}\left(P_{\text {chassis }}+n_{\text {linecard }} . P_{\text {linecard }}+\sum_{i=0}^{c} n \text { ports. } P_{r}\right. \\
&* \text { UtilizationFactors })
\end{aligned}
$$

Finally, based on the mathematical formulations above, it is easier to calculate the Energy efficient metric

Which:

Energy usage effective $=$ Facilty efficiency (Facility energy efficiency $\% *$ facility utilization $\%) *$ IT asset efficiency(IT utilization $\% *$ IT energy efficiency\%)

Where

Facility energy efficiency $\%=\frac{I T \text { load }}{\text { Total power consumed by the DC }}$

Facility Utilization $\%=\frac{\text { Actual IT load used during the process }}{\text { facility capacity }}$

IT Utilization \% is the Average CPU utilization, server utilization, etc

IT energy efficiency\% is the server, storage and network energy usage metric. 


\subsection{Proposed intelligent heuristic greedy algorithm for efficient Data Center}

Cloud data centers are composed of many computing/storage nodes (e.g. servers, CPU, memory), and network devices (network links). In order to save energy, the energy consumption of various sections in CC can be minimized. This work is focused on minimizing the energy consumption in the network links and storage nodes. This is because the links is connected by the nodes and the mobile agent is installed in the nodes.

The proposed algorithm in Figure 4 aims at reducing the number of links in the network during energy-saving periods. This is designed in such a way that the energy-savings does not in any way affect the quality of service of the cloud customers. First of all, it takes as input, the full network topology and the given traffic matrix (as generated by the customers' requests), and the threshold which is determined by the operator. The threshold is based on the maximum link utilization (MLU) of the cloud data center and should not be up to $100 \%$ of the customers' request. The idea is to make sure that energy-saving's algorithm is triggered before congestion starts. The utilization of each network link is computed (as shown in line 1), examined by the algorithm and sorted in ascending order according of respective utilizations - based on this, the removal process starts by the mobile agent. Before any link is removed, the cloud connectivity is checked (see line 5). This is to confirm that no computing node is disconnected from the cloud infrastructure. If the maximum link utilization of the entire cloud data center does not exceed the pre-defined threshold, the link can be successfully removed and scheduled for energy savings. Otherwise, the next link is checked. This continues until all the links are checked.

\section{$5 \quad$ Result evaluation and analysis}

GEANT network was used because it replicates likely scenario as the data center routing technology. The GEANT topology is an operational network in Europe for research purposes [36]. It consists 23 Point-of-Presence (PoP) nodes and 74 inter-PoP links of varying delay and bandwidth capacities. The traffic matrix was collected for a period of 2 hours in the interval of 15 minutes. During the energy savings of the network, different thresholds are considered. However, the preference of threshold is a matter of choice but will not be equal to $100 \%$. This is to give room for congestion control and avoidance during the process. More so, a synthetic topology and generated traffic matrix was used.

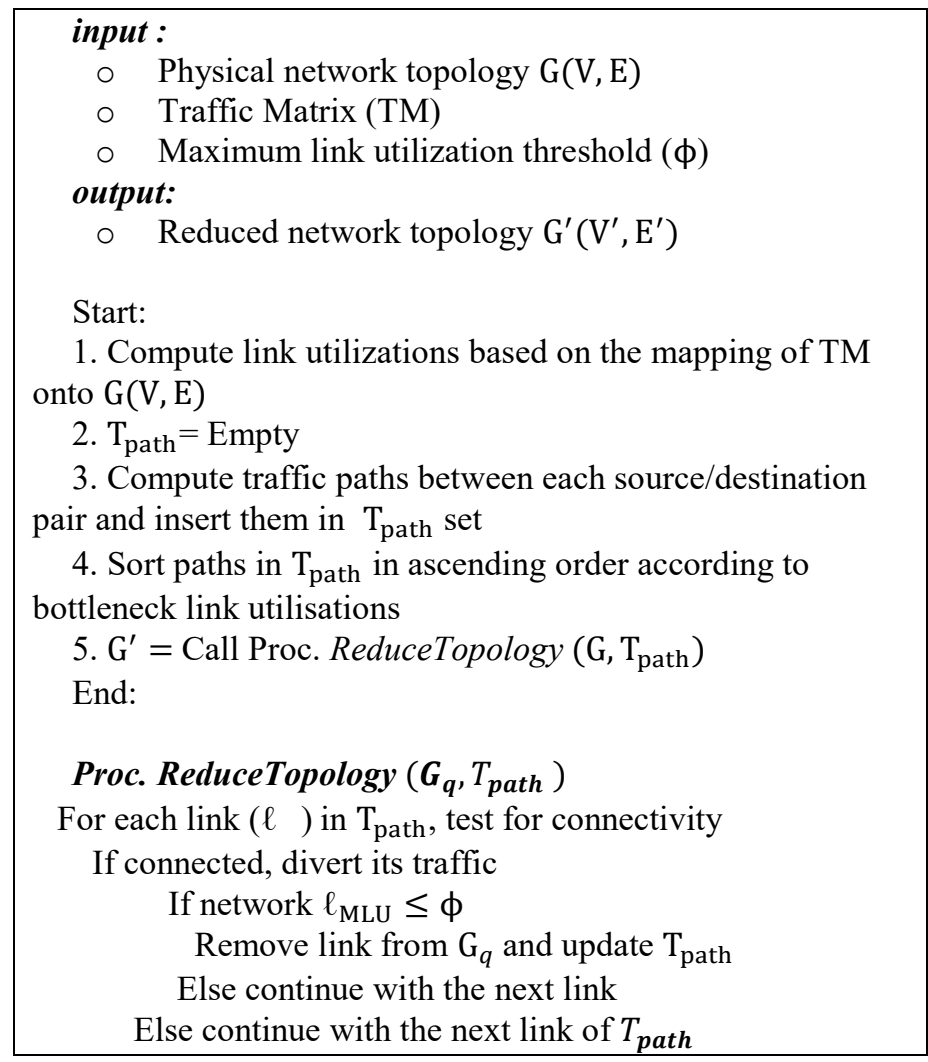

Fig. 4. Proposed intelligent agent algorithm

The experiment is setup using the traffic matrix (TM) generated by the customer's request in the GEANT network. In order to save energy in the network, the mobile agent determines the utilization of the links such that if switched-off for energy savings, it does not inhibit the performance of the network. Figure 5 and 6 shows the utilizations of different TMs. The TMs are traffics generated in every 15 minutes by the customers. As can be seen in Figure 4, there is little, or no traffic generated by links between 0 and 20. Therefore, the embedded intelligent system deems such links to be switched off. This result also shows the underutilization nature of networks. This is even worse in the next hour as shown in Figure 5 where the highest utilization is not up to $25 \%$. 


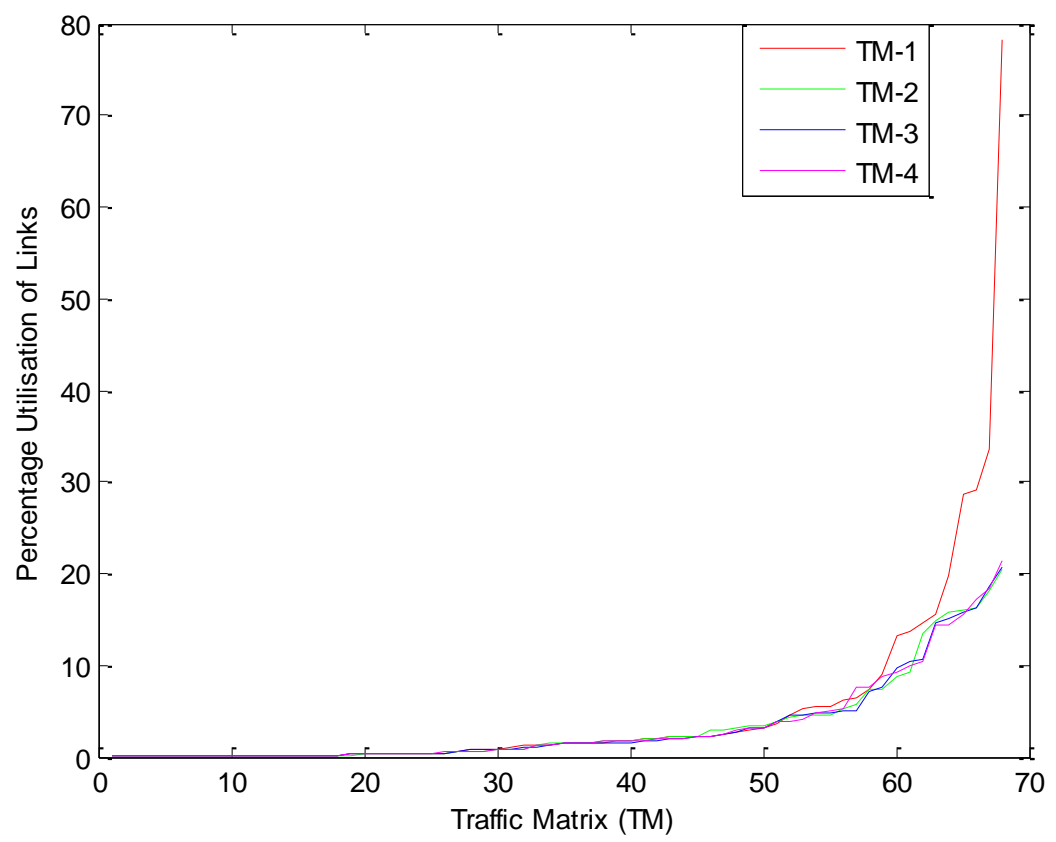

Fig. 5. Percentage utilization of each link using customers' TM.

In order to maximize the energy savings level, the embedded intelligent system also monitors the activity of the network and switches some of the links to sleeping mode or better still ON mode such that energy can be saved during those periods. As a result of this, Figure 7 shows the amount of energy saved during the 2-hour simulations. As can be seen, substantial amount of energy is saved within the two-hour traffic evaluation. This is also summarized in Table 3.

Table 3: Tabular analysis of a snapshot of a TM

\begin{tabular}{|l|l|l|l|}
\hline Traffic & Links & MLU & ALU \\
\hline TM1 & 32 & 78.16 & 5.08 \\
\hline TM2 & 33 & 77.72 & 5.57 \\
\hline TM3 & 32 & 80.67 & 5.62 \\
\hline TM4 & 34 & 81.28 & 5.71 \\
\hline TM5 & 31 & 82.31 & 5.77 \\
\hline TM6 & 31 & 85.21 & 5.86 \\
\hline TM7 & 32 & 84.27 & 5.78 \\
\hline TM8 & 31 & 81.23 & 5.88 \\
\hline
\end{tabular}

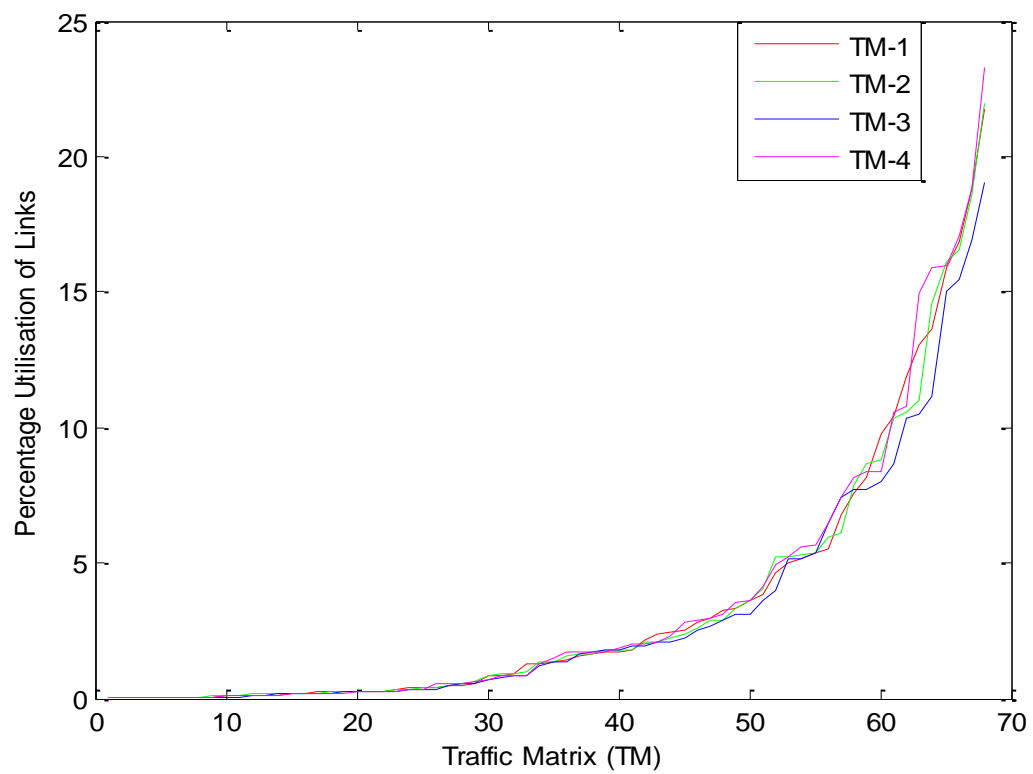

customers' TM for the next hour.

Fig. 6. Percentage utilization of each link using 


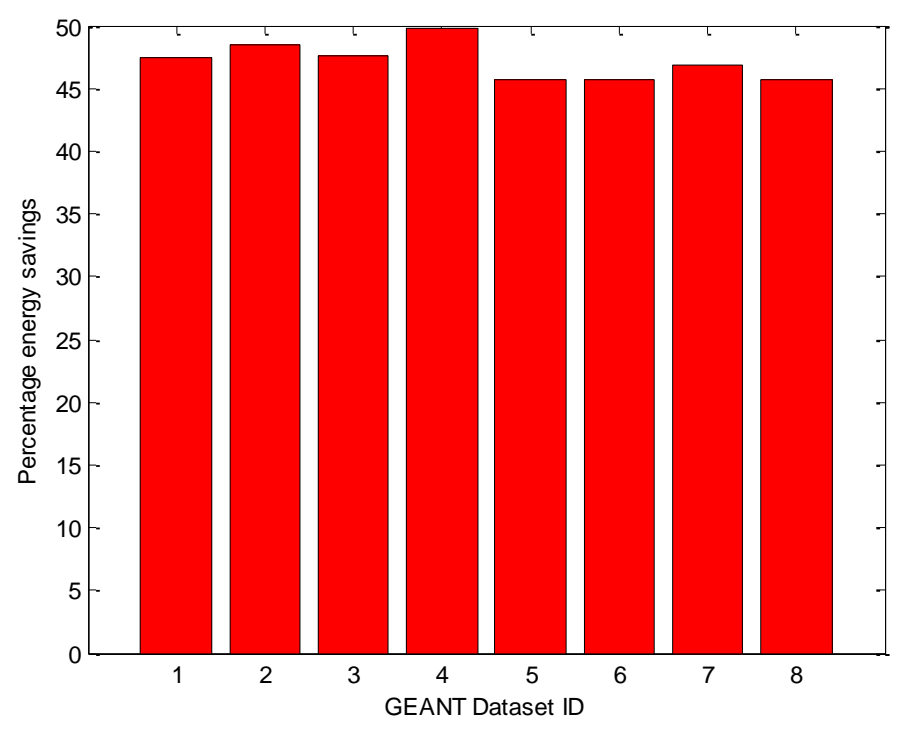

Fig. 7. Graph of percentage energy savings of GEANT network.

According various researches, there is high rate of underutilization of cloud platform. With the introduction of an intelligent system, it leverages the complexity of cloud environment by having full migration access to all the cloud components without disrupting the quality of service or bridging the service level agreement. That is why my proposed idea can never be overlooked. It balances the trade-off between efficiency in performance vs power usage in the cloud environment. Importantly this proposed work will be the first of its kind to monitor and manage the power usage during and after scheduling process involving the link, node and switches. This approach considers all the cloud components (i.e. the network, links, switches and traffic method) in saving energy in the cloud environment. It will efficiently manage, monitor and aid proper scheduling process with less response time and human error. This will therefore propel more energy efficient cloud data center and cloud environment at large.

\section{Conclusion and future work}

Energy consumption has become a major research interest to the CC community as a result of unprecedented rise in ICT sector. As Internet keeps advancing in different technologies, there is always a greater increase in the rate of end-users which leads to high energy consumption. This work piece is centered on developing an online approach to mitigating the high rate of energy consumption in CC. Unlike normal norms, energy can be saved in CC using offline approach i.e. an approach that is centered on night time or when traffic is less. However, such approach does not adapt to the dynamics of the data center network scheme which enable client access on 24 hours bases. In this paper, an embedded intelligent sensor was proposed in order to monitor the activities of the data center focusing on aggregation layer. During low traffic in the network, some activities are shut down for energy savings or put in sleeping mood. This is a novel approach to energy savings since it does not need to store/know the statistics of the network and also execute task on timely basis with less bottlenecks like pervious works. Therefore, the introduction of this approach achieves a greater energy savings compared to other existing schemes.

Future work will continue to investigate on the best possible method to reducing the operational cost of running the cloud system by minimizing the DC energy usage. This will greatly encourage smart cities actualization because it will empower both the vulnerable and medium scale enterprise toward using cloud innovation which is bedrock of internet of thing.

\section{References}

1. Stott, I., Sanders, D.: A new prototype intelligent mobility system to assist powered wheelchair users. Industrial Robot 26 (6), $466-475$ (1999).

2. Goodwin, MJ., Sanders DA., Poland GA.: Navigational assistance for disabled wheelchair-users. Euromicro Conference 95 Volume: 43 73-79 (1997).

3. Stott, I., Sanders, D.: New powered wheelchair systems for the rehabilitation of some severely disabled users. Int' Journal of Rehabilitation Research 23 (3), 149-153 (2000).

4. Stott, I., Sanders, D.: The use of virtual reality to train powered wheelchair users and test new wheelchair systems. Int Journal of Rehab Research 23 (4), 321-326. (2000).

5. Sanders, DA., Bausch, N.: Improving Steering of a Powered Wheelchair Using an Expert System to Interpret Hand Tremor. Proc of Intelligent Wheelchairics and Applications (Icira 2015), Pt Ii, vol. 9245, pp. 460-471 (2015).

6. Sanders, DA.: Using self-reliance factors to decide how to share control between human powered wheelchair drivers and ultrasonic sensors, IEEE Transactions on Neural Systems and Rehabilitation Engineering, vol. 25, no. 8, pp. 1221-1229 (2017).

7. Ma,C., Li,W., Cao, J., Gravina, R., Fortino, G.: Cloud-Based Wheelchair Assist System for Mobility Impaired Individuals. In: Li W. et al. (eds) Internet and Distributed Computing Systems. Lecture Notes in Computer Science 9864, Springer, pp 107-118 (2016).

8. Fortino, G., Giannantonio, R., Gravina, R., Kuryloski, P., Jafari, R.: Enabling effective programming and flexible management of efficient body sensor network applications. IEEE Trans. Hum.-Mach. Syst. 43(1) 115-133 (2013). 
9. Gravina, R., Andreoli, A., Salmeri, A., Buondonno, L., Raveendranathan, N., Loseu, V., Giannantonio, R., Seto, E., Fortino, G.: Enabling multiple BSN applications using the SPINE framework. Proc' International Conf' on Body Sensor Networks 228-233 (2010).

10. Fortino, G., Fatta, G., Pathan, M., Vasilakos, A.: Cloud-assisted body area networks: state-of-the-art and future challenges. Wirel. Netw. 20(7), 1925-1938 (2014)

11. Sanders, D., Langner, M., Tewkesbury, GE.: Improving wheelchair-driving using a sensor system to control wheelchair-veer and variable-switches as an alternative to digitalswitches or joysticks. Industrial Robot 37 (2), 157-167 (2010).

12. Sanders, D., Tewkesbury, GE., Stott, IJ., Robinson, DC.: Simple expert systems to improve an ultrasonic sensor-system for a tele-operated mobile-robot. Sensor Review 31 (3) 246-260 (2011).

13. Sanders, DA., Graham-Jones, J., Gegov, A.: Improving ability of tele-operators to complete progressively more difficult mobile robot paths using simple expert systems and ultrasonic sensors. Industrial Robot-an International Journal 37 (5), pp: 431-440. (2010).

14. Sanders, DA.: Non-Model-Based Control of a Wheeled Vehicle Pulling Two Trailers to Provide Early Powered Mobility and Driving Experiences. IEEE Transactions on Neural Systems and Rehabilitation Engineering 26 (1), 96-104 (2018).

15. Sanders, D., Gegov, A.: Using artificial intelligence to share control of a powered-wheelchair between a wheelchair user and an intelligent sensor system, EPSRC (2018).

16. Sanders, D.: Comparing ability to complete simple tele-operated rescue or maintenance mobile-robot tasks with and without a sensor system. Sensor Review 30(1), 40-50 (2010).

17. Sanders, DA., Ndzi,, D., Chester, S., Malik, M.: Adjustment of Tele-Operator Learning When Provided with Different Levels of Sensor Support While Driving Mobile Robots. Proceedings SAI Intelligent Systems Conference 2016, Vol 2 -16, 548-558 (2018).

18. Sanders, D.: Environmental sensors and networks of sensors. Sensor Review 28 (4), 273-274 (2008).

19. Sanders, D.: Analysis of the effects of time delays on the teleoperation of a mobile robot in various modes of operation. Industrial Robot 36 (6), $570-584$ (2009).

20. Sanders, D.: Viewpoint - Force sensing. Industrial Robot 34(4), pg 177. 268 (2007).

21. Sanders, DA., Sanders, HM., Gegov, A., Ndzi, D.: Rule-Based System to Assist a Tele-Operator with Driving a Mobile Robot. Proc' SAI Intelligent Systems Conference (Intellisys) 2016, Vol 2 - 16, 599-615 (2018).

22. Sanders, DA., Gegov, A., Ndzi, D.: Knowledge-based expert system using a set of rules to assist a tele-operated mobile robot, in Studies in Computational Intelligence, (eds) Y. Bi, S. Kapoor, and R. Bhatia, 2018, 751, Springer, 371-392 (2018).

23. Fortino, G., Guerrieri, A., Bellifemine, F., Giannantonio, R.: SPINE2: developing BSN applications on heterogeneous sensor nodes. In: IEEE International Symposium on Industrial Embedded Systems, 128-131 (2009).

24. Fortino, G., Guerrieri, A., Bellifemine, F., Giannantonio, R.: Platform-independent development of collaborative wireless body sensor network applications: SPINE2. In: IEEE International Conference on Systems, Man and Cybernetics, 3144-3150 (2009)

25. Yang, H., Tate, M.: A descriptive Literature Review and Classification of Cloud Computing Research, Communications of the Association for Information Systems 31 , Article 2.

26. Mell, P. and Grance, T, "The NIST Definition of Cloud Computing" National Institute of Standards and Technology, [Online]. Available at: http://csrc.nist.gov/publications/drafts/800-145 (2009).

27. https://www.businessnewsdaily.com/4982-cloud-vs-data-center.html

28. Li B., et al.: EnaCloud: an energy-saving application live placement approach for cloud computing environments, Proc of international conf on cloud computing (2009).

29. Pelley, S.: Understanding and abstracting total data centre power (2009)

30. Aschberger, C.,Halbrainer, F.: Energy efficiency in cloud computing “venue July 18, (2013).

31. Brown, R.: Report to congress on server and data centre energy efficiency public law 109-431,” U.S. Environ. Protection Agency, Washington, DC, USA (2007).

32. Koomey, J.: Growth in Data Centre Electricity Use 2005 to 2010. Oakland, CA, USA: Analytics Press (2011).

33. Meijer, G.: Cooling energy-hungry data centres: Science, vol. 328, no. 5976, pp. 318-319, Apr. [9] G. Group, forecast: Data centres, worldwide, 2010-2015, Accessed (2010).

34. Fan, X.: W.-D. Weber, and L. A. Barroso, "Power provisioning for a warehouse-sized computer," in ISCA '07: Proceedings of the 34th annual international symposium on Computer architecture (2007).

35. Mahadevan, D. Sharma, P., Banerjee, P.: A power benchmarking framework for network devices. In Proceeding of the 8th international IFIP-TC6 networking conference Germany,11-15 (2009)

36. Song, SL., Barker, K., Kerbyson, D.: Unified Performance and power modelling of scientific workloads in Pro. 1st workload E2SC, 4:1-4:8 (2013).

37. Amaldi, E., Capone, A., Gianoli, LG.: Energy-Aware IP Traffic Engineering with Shortest Path Routing" Comput. Network (2013).

38. Hlavacs, H., Costa, G.m Pierson, J.: Energy Consumption of Residential and Professional Switches, Proc of the IEEE International Conference on Computational Science and Engineering, 240-246 (2009).

39. Forster, C., Dickie, L., Maile, G., Smith, H., Crisp, M.: Understanding the environmental impact of communication systems, OFCOM (2009).

40. Chiaraviglio, L., Mellia, M.., Neri, F.: Energy-aware backbone networks: A case study, Proc' of IEEE International Conference on Communications 1-5 (2009).

41. Adelin, A.. Owezarski P., Gayraud, T.: On the Impact of Monitoring Router Energy Consumption for Greening the Internet, IEEE/ACM International Conference on Grid Computing (2010)

42. Zhang, Q., Cheng, L., Boutaba, R.: Cloud computing: state-of-the-art and research challenges" Published online: The Brazilian Computer Society (2010).

43. Arya, L.K., Verma, A.: Workflow scheduling algorithms in cloud environment- A Survey, Recent Advances in Engineering and Computational Sciences, IEEE,1-4 (2014).

44. Aschberger, CFranziska F.: Energy Efficiency in Cloud Computing (2013).

45. Luo, L., Wu, W., Dichen D., Fei, DZ., Yan, Y., Mao, M.: A resource scheduling algorithm of cloud computing based on energy efficient optimization methods, Green Computing Conference (IGCC), 1-6 (2012).

46. Gunaratne, C., Christensen, K., Suen, S.: Ethernet Adaptive Link Rate (ALR): Analysis of a Buffer Threshold Policy, IEEE GLOBECOM (2006).

47. Nedevschi, S., Popa, L., Iannaccone, G., Ratnasamy, S., Wetherall, D.: Reducing Network Energy Consumption via Sleeping and Rate-Adaptation, Proc. of the $5^{\text {th }}$ USENIX NSDI, pp. 323-336 (2008).

48. Huang, Q., Su, S., Li, J., Xu, P., Shuang, K., Huang,X.: Enhanced Energy-Efficient Scheduling for Parallel Applications in Cloud, Proc of $12^{\text {th }}$ EEE/ACM International Symposium on Cluster, Cloud and Grid Computing, CCGRID '12, 781-786, (2012).

49. Liu, J., Zhao, F., Liu, X., He, W.: Challenges Towards Elastic Power Management in Internet Data Centers. Proc $2^{\text {nd }}$ international workshop on cyber-physical systems (WCPS), in conjunction with ICDCS Montreal, Canada, (2009).

50. Zhang, M., Yi, C., Liu, B., Zhang, B.: GreenTE: Power-Aware Traffic Engineering, Proc. IEEE International Conference on Network Protocols (2010).

51. Berl, A., Gelenbe, E., di Girolamo, M., Giuliani, G., de Meer, H., Quan Dang, M., Pentikousis, K.: Energy-Efficient Cloud Computing. Oxford University Press on behalf of The British Computer Society (2009).

52. Aschberger, C., Halbrainer, F.: Energy efficiency in cloud computing (2013).

53. Low, C., Chen, Y.: Understanding the determinants of cloud computing adoption, Industrial Management \& Data Systems 111 (7). (2011).

54. Lal Sahu, B., Tiwari, R.: International Journal of Advanced Research in Computer Science and Software Engineering 2 (9) (2012).

55. Mattew, T., Sekaran, C., Jose, J.: Study and Analysis of Various Task Scheduling Algorithm in the Cloud Computing environment, ICACCI International Conference (2014)

56. Tracy, D., Howard, B., Siegel, J., Beck, N.: A Comparison of Eleven Static Heuristics or mapping a Class of Independent Tasks onto Heterogeneous Distributed Computing Systems, Journal of Parallel and distributed Computing 61, 810-837, (2001).

57. Nagadevil, S., Satyapriya, K., Malathy, D.:A Survey on Economic Cloud Schedulers for Optimized Task Scheduling Algorithm, International Journal of Advance Engineering Technology (2013).

58. Cao, J., Spooner, D., Jarvis, SA., Nudd, GR., Grid Load Balancing Using Intelligent Agents, Future Generation Computer System 21 (1) 135-149 (2005).

59. Izakian, H., Abraham, A., Snasel, V.: Comparison of Heuristics for Scheduling Independent Tasks on Heterogeneous Distributed Environments, Proc of International Joint Conference on Computational Sciences and Optimization, IEEE, Vol 1 (2009).

60. The GEANT Network: http://www.geant.net 